\title{
First-principles investigation of hyperfine interactions for nuclear spin entanglement in photo-excited fullerenes
}

\author{
Vasileia Filidou, ${ }^{1,2}$ Davide Ceresoli, ${ }^{1,3}$ John J. L. Morton, ${ }^{1,2}$ and Feliciano Giustino ${ }^{1}$ \\ ${ }^{1}$ Department of Materials, University of Oxford, Parks Road, Oxford OX1 3PH, United Kingdom \\ ${ }^{2}$ CAESR, Clarendon Laboratory, Oxford University, Oxford, OX1 3PU, United Kingdom \\ ${ }^{3}$ CNR-ISTM, via Golgi 19, 20133 Milan, Italy
}

(Dated: July 3, 2018)

\begin{abstract}
The study of hyperfine interactions in optically excited fullerenes has recently acquired importance within the context of nuclear spin entanglement for quantum information technology. We here report a first-principles pseudopotential study of the hyperfine coupling parameters of optically excited fullerene derivatives as well as small organic radicals. The calculations are performed within the gauge-invariant projector-augmented wave method [C. Pickard and F. Mauri, Phys. Rev. B. 63, 245101 (2001)]. In order to establish the accuracy of this methodology we compare our results with all-electron calculations and with experiment. In the case of fullerene derivatives we study the hyperfine coupling in the spin-triplet exciton state and compare our calculations with recent electron paramagnetic resonance measurements [M. Schaffry et al., Phys. Rev. Lett. 104, 200501 (2010)]. We discuss our results in light of a recent proposal for entangling remote nuclear spins in photo-excited chromophores.

PACS numbers: 33.15.Pw, 31.30.Gs, 71.20.Tx
\end{abstract}

\section{INTRODUCTION}

In the area of quantum information processing substantial efforts are being devoted to exploit the magnetic properties of molecular systems in order to realize quantum logic operations $\frac{1}{=}$ In a recent proposal for entangling two nuclear spins using a photo-excited transient electron spin, $\stackrel{2}{=}$ the strength of the hyperfine interaction (HFI) has been identified as a key parameter determining the entanglement efficiency. These investigations raise the question on how to optimize such parameters, and how to design new materials with tailored magnetic properties for specific quantum logic operations.

First-principles calculations based on densityfunctional theory (DFT) have recently met with a number of successes in the calculation of the magnetic properties of materials, in particular when it comes to the building blocks of the spin Hamiltonian such as chemical shifts, $\stackrel{3-8}{-\frac{8}{2}}$ J-couplings $, 9,10$ and g-tensors $\stackrel{11.12}{=}$ As magnetic spin resonance experimental techniques become more accurate, and novel applications to physics, biology, and materials science are being explored, DFT-based modelling of charge/spin interactions hold the promise of becoming a key player in the interpretation of measured magnetic responses and in the design of materials with tailored functionality.

In this work we aim to establish the validity and accuracy of DFT-based modelling in the calculation of HFI parameters in a class of materials of interest for quantum information technology. We here focus on the HFI interactions in the fullerene derivatives synthesized in Ref. 2, since electron spin resonance measurements on these materials have already been performed.

The manuscript is organized as follows. In Sec. [I] we briefly review the connection between the HFI parameters and the efficiency of the quantum entanglement op- erations introduced in Ref. 2. In Sec. III we describe the computational methodology that we employ for calculating hyperfine tensors. In Sec. IVA we discuss our HFI calculations for a series of small radicals. In this section we establish the accuracy and reliability of pseudopotential (PP) calculations of the HFI by comparing with all-electron (AE) calculations and with experiment. In Sec. IVB we present our calculations of the HFI on photo-excited fullerene derivatives, and we discuss our results in light of the entanglement protocol proposed in Ref. 2. In Sec. $\mathrm{V}$ we present our conclusions.

\section{ENTANGLING NUCLEAR SPINS VIA THE HYPERFINE INTERACTION}

In Ref. 2 a quantum information processing protocol involving nuclear spins has been introduced. This protocol involves the interaction of two nuclear spins attached to a fullerene molecule through functional additions carrying isotopically labeled nuclei. The ground state of this fullerene derivative is spin-unpolarized, and hence the HFI of the nuclear spins is vanishing. Upon optical excitation a spin-triplet excitonic state is generated in the molecule, and the nuclear spins interact with the spinpolarized electrons leading to a non-vanishing hyperfine coupling. As a result the two nuclear spins experience an interaction which is mediated by the spin of the exciton. Such interaction can be used to perform an entanglement operation between the two nuclear spins. When the exciton decays and the fullerene reverts to its spinunpolarized ground state, the nuclear spins may remain entangled. The efficiency of this protocol results from the competition between three timescales: (i) the time required to perform the entanglement operation, (ii) the exciton lifetime, and (iii) the decoherence time of the en- 
tangled state ${ }^{2}$ The competition between these timescales defines an optimum range of HFI strength for achieving maximum entanglement.

In order to establish the feasibility of the protocol proposed in Ref. 2, pulsed electron paramagnetic resonance experiments were performed on a prototype system, the fullerene diethyl malonate molecule $\left[\mathrm{C}_{60}-\mathrm{C}_{7} \mathrm{H}_{12} \mathrm{O}_{4}\right.$, system M1 in Fig. 1(a)]. Although this system carries only one isotopically labeled nuclear spin associated with the ${ }^{13} \mathrm{C}$ atom of the functional group directly bonded to the cage, it was possible to extract relevant information such as the lifetimes of the exciton states and the HFI parameters. For the system considered the measured relaxation rates of $90-100 \mu$ s at $20-50 \mathrm{~K}$ correspond to maximum entangling power when the HFI parameters are in the range $2 \mathrm{MHz}$ to $12 \mathrm{MHz}$, with an optimal value of $6 \mathrm{MHz} .2$ The measured HFI parameter of $3 \mathrm{MHz}$ in this prototype system with a single nuclear spin falls within the optimum theoretical range and motivates further work in this area.

The key open question which remains is how to identify systems involving multiple nuclear spins and exhibiting optimal HFI strengths. One possible route for answering such question is to employ first-principles materials modelling based on density-functional theory in order to screen candidate systems prior to their experimental synthesis. Accordingly, in the present work we aim at establishing the accuracy of such first-principles calculations, in view of optimizing and designing functional materials for spin-based quantum information processing.

\section{COMPUTATIONAL METHODOLOGY}

\section{A. Hyperfine tensor from first principles}

The coupling of the electron spin $\vec{S}$ to a set of $N$ nuclear spins $\vec{I}_{l}(l=1, \ldots N)$ can be described using the following hyperfine Hamiltonian:15

$$
\hat{H}=\sum_{l=1, N} \vec{S}^{T} \mathbf{A}^{(l)} \vec{I}_{l}
$$

$\mathbf{A}^{(l)}$ being the hyperfine coupling tensor associated with the $l$-th nucleus at the site $\mathbf{R}_{l}$. In atomic units the hyperfine tensor can be written as: ${ }^{15} A_{i j}^{(l)}=a^{(l)} \delta_{i j}+b_{i j}^{(l)}$, with

$$
a^{(l)}=\frac{8 \pi}{3} g_{e} \mu_{e} g_{l} \mu_{N} \rho_{\mathrm{s}}\left(\mathbf{R}_{l}\right)
$$

and

$$
b_{i j}^{(l)}=g_{e} \mu_{e} g_{l} \mu_{N} \int d \mathbf{r} \frac{3 r_{i} r_{j}-r^{2} \delta_{i j}}{r^{5}} \rho_{\mathrm{s}}(\mathbf{r}) .
$$

In these equations $\rho_{\mathrm{S}}(\mathbf{r})=\rho_{\uparrow}(\mathbf{r})-\rho_{\downarrow}(\mathbf{r})$ is the electron spin density, $g_{e}$ the free-electron g-factor, $\mu_{e}$ the Bohr magneton, $g_{l}$ the gyromagnetic ratio of the $l$-th nucleus and $\mu_{N}$ the nuclear magneton. In Eq. (3) $\mathbf{r}$ is relative to the nuclear site $\mathbf{R}_{l}$ and $r=|\mathbf{r}|$. The component $a^{(l)}$ of the tensor $\mathbf{A}^{(l)}$ provides the isotropic hyperfine interaction and is referred to as the Fermi contact term. The traceless tensor $b_{i j}^{(l)}$ gives the anisotropic or dipolar hyperfine interaction. The anisotropic tensor $b_{i j}^{(l)}$ is conventionally given in terms of its eigenvaules $b_{x x}^{(l)}, b_{y y}^{(l)}$, and $b_{z z}^{(l)}$. We note that the principal axis of the hyperfine tensor $\mathbf{A}^{(l)}$ and of its anisotropic part $b_{i j}^{(l)}$ do coincide by construction.

The evaluation of the HFI parameters using Eqs. (2) and (3) requires the calculation of the electron spin density. In the dipole-dipole term $b^{(l)}$ the electron spindensity is weighted by the factor $r^{-3}$ and the contributions outside of the core region are dominant. In the Fermi contact term the electron spin density must be evaluated at the position of the nucleus, therefore an accurate description of the electron density within the core region is essential. While standard pseudopotential methods can be used for calculating the dipole-dipole term $b^{(l)}$, the calculation of the Fermi contact contribution requires the use of all-electron methods or the projected augmented wave (PAW) method. 15 The PAW method enables the reconstruction of the all-electron wavefunction inside the core region, starting from a pseudopotential calculation of the valence electronic structure, and has successfully been applied to the calculation of hyperfine parameters. 16

\section{B. Systems considered}

In Ref. 2 we reported a preliminary calculation of the isotropic component of the HFI tensor of the M1 system [Fig. 1(a)], and of a related fullerene derivative carrying two ${ }^{13} \mathrm{C}$ nuclear spins [system B1 in Fig. 1(b)]. Here we complement the work of Ref. 2 by calculating the entire HFI tensor of these systems, and we further extend it to fullerene derivatives of various conformations. Furthermore, we perform calculations on a number of small open-shell molecules, both within $\mathrm{PP}$ and AE methods, in order to establish the accuracy of the computational methodology.

We consider following small molecules containing hydrogen and oxygen: $\mathrm{CH}_{2} \mathrm{CH}_{3}, \mathrm{CH}_{2} \mathrm{CH}, \mathrm{CO}_{3}, \mathrm{H}_{3} \mathrm{CO}$, $\mathrm{HCO}, \mathrm{H}_{2} \mathrm{CO}^{+}$, and $\mathrm{CH}_{3}$. In the case of diethyl malonate fullerene derivatives we consider the mono-adduct M1 [Fig. 1(a)], as well as the three regioisomeric bisadducts B1-B3 shown in Fig. 1(b). During the synthesis of stable regioisomeric bis-adducts from diethyl malonate and fullerene molecules, the trans- 1 isomer [model B1 in Fig. 1(b)] is produced with the lowest yield (0.8\%), while the equatorial and the trans-3 isomers [models B2 and B3 in Fig. 1(b), respectively] are produced with the highest yields ( $16 \%$ and $12 \%$, respectively) 17 


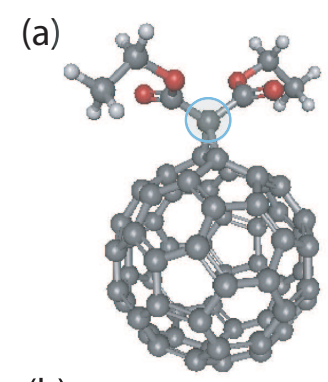

(b)

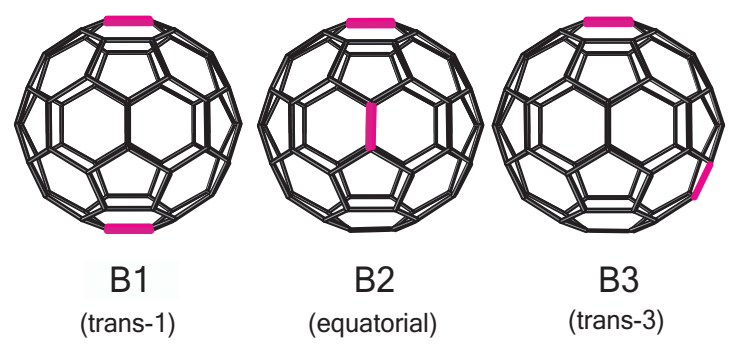

FIG. 1: (a) Ball-and-stick (left) and schematic (right) representations of the diethyl malonate $\left(\mathrm{C}_{7} \mathrm{H}_{12} \mathrm{O}_{4}\right) \mathrm{C}_{60}$ monoadduct (M1). The bridging ${ }^{13} \mathrm{C}$ atom carrying a nuclear spin is indicated. In the schematic representation the red bond indicates the anchoring point of the adduct on the fullerene cage. Atomic color code: C (grey), O (red), H (white). (b) Schematic representations of three regioisomeric diethyl malonate fullerene bis-adducts (B1-B3).

\section{Calculations details}

We perform pseudopotential (PP) density-functional theory calculations within the generalized gradient approximation of Perdew, Burke, and Ernzerhof (PBE) $\stackrel{18}{\underline{18}}$ We describe the valence electronic wavefunctions using a planewaves basis set with a kinetic energy cutoff of 70 Ry. We take into account the core-valence interaction using Troulier-Martins normconserving pseudopotentials $\underline{19,20}$ For the calculation of the HFI tensor we use the gaugeincluding projector-augmented wave method (GIPAW) of Ref. 21. We use periodic simulation cells, and in order to minimize interactions between periodic replicas of the molecules, we use cells of size $12 \AA$ for the small molecules, and cells of size up to $24 \AA$ for the fullerene derivatives. All PP calculations are performed using the quantum-ESPRESSO software package $\underline{\underline{25}}$

For the calculation of the Fermi contact term, the accurate description of the electronic wavefunctions in the core region is required. In pseudopotential methods only valence electrons are described, and in some cases this might lead to significant errors due to the neglect of core polarization effects. Core polarization refers to the spin polarization of core electrons induced by the spin of valence electrons, and leads to an additional contribution to the Fermi contact term, $\stackrel{22,23}{2}$ Within PP calculations we introduce core polarization corrections using the perturbative approach of Ref. 24 as follows. For each atom with core electrons $(\mathrm{C}$ and $\mathrm{O})$ we calculate the spherical average of the spin density around the nuclues up to a radius of 5 a.u. using a logarithmic radial grid. This average is used to evaluate the perturbation $\Delta V$ due to valence polarization following Eq. (20) of Ref. 24, and subsequently the contribution to the Fermi contact term arising from the core polarization using Eq. (17) of Ref. 24. The atomic all-electron wavefunctions are stored in the pseudopotentials files and the integrations are carried out on the radial grid. In the following, pseudopotential calculations including the core polarization correction will be referred as "PP-CP", while those without core polarization will be denoted simply by "PP". In the case of light atoms such as those considered in this work relativistic effects are expected to be negligible, therefore we generated the pseudopotentials by solving the nonrelativistic Schrödinger equation (it is custom practice to solve the scalar-relativistic equation only for atoms starting from the fourth row of the periodic table). In evaluating the Fermi contact term, the first relativistic correction can be taken into account through a spherical integration of the spin density up to the Thomson radius $r_{T}$, which provides an estimate of the finite size of the nucleus. However, the Thomson radii of $\mathrm{H}$ and $\mathrm{C}$ fall around the first point of our pseudopotential radial grid, therefore it seems more appropriate to evaluate the spin density at the nucleus (this is done by extrapolating the three innermost values on the grid).

In order to establish the accuracy of PP calculations of HFI parameters we also perform AE calculations using the PBE exchange and correlation, as implemented in the Gaussian 09 program package $\stackrel{26}{\underline{2}}$ For the AE calculation of the HFI tensor we adopt the EPR-II basis, which has been purposely designed to accurately describe the Fermi contact term. $\underline{\underline{27}}$

In order to study the HFI in optically-excited fullerene derivatives we perform calculations using the ground state of the $S_{z}=1$ electron spin configuration. In fact, while density-functional theory does not describe correctly excited states, the lowest spin-triplet state of $\mathrm{C}_{60}$, with the HOMO and LUMO states singly occupied with parallel spins, is the ground state of the $S_{z}=1 \mathrm{spin}$ configuration, hence the use of DFT is legitimate 28,29

For each structure M1-B3 shown in Fig. 1 we optimize several initial geometries in both the singlet $S_{z}=0$ and triplet $S_{z}=1$ configurations in order to identify the corresponding ground state. The energy difference between the triplet and the singlet states ranges from 1.29 to 1.33 $\mathrm{eV}$ across all the models considered. This result is in agreement with the measured excitation energy of 1.30 $\mathrm{eV}$ of the non-functionalized fullerene $\mathrm{C}_{60} \stackrel{44.45}{\stackrel{4}{*} \text { This sug- }}$ gests that our description of the lowest triplet exciton state is adequate. 
TABLE I: Fermi contact hyperfine coupling parameters of small molecules containing $\mathrm{C}$, and $\mathrm{H}$. We report calculations using the GIPAW method without accounting for corepolarization effects (PP), calculations within GIPAW including core-polarization corrections (PP-CP), all-electron calculations (AE), and measured Fermi contact parameters. The atoms are listed according to corresponding chemical formula.

\begin{tabular}{llcccc}
\hline \hline Molecule Atom & \multicolumn{4}{c}{ Fermi contact term $(\mathrm{MHz})$} \\
& & PP & PP-CP & AE & Expt. \\
\hline $\mathrm{CH}_{2} \mathrm{CH}$ & $\mathrm{C}(1)$ & 397 & 305 & 306 & $302^{\mathrm{a}}$ \\
& $\mathrm{H}(1)$ & 125 & & 124 & 96 \\
& $\mathrm{H}(2)$ & 191 & & 190 & 192 \\
& $\mathrm{C}(2)$ & -27 & -17 & -18 & -24 \\
& $\mathrm{H}(3)$ & 38 & & 43 & 37 \\
\hline $\mathrm{CH}_{2} \mathrm{CH}_{3}$ & $\mathrm{C}$ & 205 & 81 & 79 & $83^{\mathrm{b}}$ \\
& $\mathrm{H}$ & -63 & & -61 & -69 \\
& $\mathrm{C}$ & -40 & -38 & -34 & -38 \\
& $\mathrm{H}$ & 51 & & 40 & 75 \\
\hline $\mathrm{CH}_{3}$ & $\mathrm{C}$ & 214 & 79 & 72 & $80^{\mathrm{g}}$ \\
& $\mathrm{H}$ & -69 & & -66 & -69 \\
\hline $\mathrm{H}_{3} \mathrm{CO}$ & $\mathrm{H}$ & 94 & & 95 & 122 \\
& $\mathrm{C}$ & -35 & -44 & -37 & $-44^{\mathrm{d}}$ \\
\hline $\mathrm{HCO}^{2}$ & $\mathrm{H}$ & 379 & & 367 & 356 \\
& $\mathrm{C}$ & 435 & 428 & 389 & $367^{\mathrm{e}}$ \\
\hline $\mathrm{H}_{2} \mathrm{CO}+$ & $\mathrm{H}$ & 279 & & 372 & 372 \\
& $\mathrm{C}$ & -76 & -83 & -87 & $-109^{\mathrm{f}}$ \\
\hline $\mathrm{CO}_{3}$ & $\mathrm{C}$ & -31 & -31 & -28 & $-32^{\mathrm{c}}$ \\
\hline \hline
\end{tabular}

${ }^{\mathrm{a}}$ Refs. 30.31,

${ }^{\mathrm{b}}$ Ref. 32,

${ }^{\mathrm{c}}$ Ref. 33 ,

${ }^{\mathrm{d}}$ Ref. 34,35 ,

${ }^{\mathrm{e}}$ Ref. 36 ,

${ }^{\mathrm{f}}$ Ref. 37,

${ }^{\mathrm{g}}$ Ref. 38.

\section{RESULTS AND DISCUSSION}

\section{A. HFI parameters of small molecules}

In Table 1 we present the results of our pseudopotential calculations and we compare with all-electron calculations and with experiment. For a quantitative analysis we present both pseudopotential results with (PP-CP) and without (PP) core polarization correction.

In the cases of $\mathrm{CH}_{2} \mathrm{CH}, \mathrm{CH}_{2} \mathrm{CH}_{3}$, and $\mathrm{CH}_{3}$ Table I shows that the neglect of core polarization for the $\mathrm{C}$ atoms leads to an underestimation of the Fermi contact term by as much as $92-135 \mathrm{MHz}$. When the core polarization correction is introduced, all the calculations tend to agree rather well with our AE results and with experiment. In the case of $\mathrm{H}$ atoms, where the core and valence do coincide, our PP results are in good agreement with
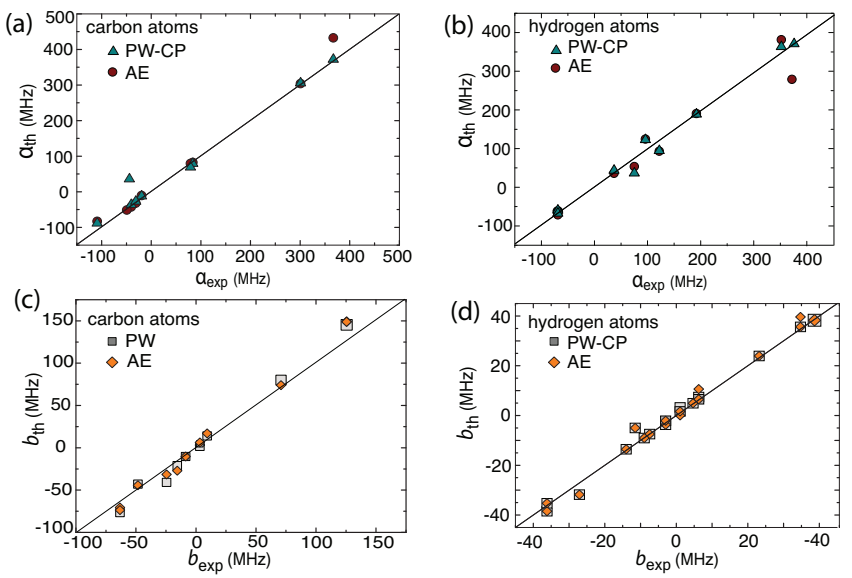

FIG. 2: Comparison between the HFI parameters calculated using PP-CP and AE methods and experimental data, from Tables \and П (a) Fermi contact term of $\mathrm{C}$ atoms. (b) Fermi contact term of $\mathrm{H}$ atoms. (c) Dipolar coupling of $\mathrm{C}$ atoms. (d) Dipolar coupling of $\mathrm{H}$ atoms. If the calculations were able to predict experimental data exactly, then all the data points would fall on the diagonal solid line.

AE and experimental data.

Figures 2(a),(b) show a comparison between the Fermi contact term for $\mathrm{C}$ and $\mathrm{H}$ atoms calculated with the PP$\mathrm{CP}$ method or the AE method, and experiment. The relative accuracy of $\mathrm{PP}-\mathrm{CP}$ calculations and $\mathrm{AE}$ calculations in the case of $\mathrm{C}$ atoms appears similar for this set of test molecules. Indeed the largest deviations from experiment are of $17 \%$ and $13 \%$ for PP-CP and AE calculations, respectively. In absolute terms the largest deviations from experiment are $61 \mathrm{MHz}(22 \mathrm{MHz}$ ) for $\mathrm{C}$ atoms using PP-CP (AE) calculations, and $93 \mathrm{MHz}(28$ $\mathrm{MHz}$ ) for $\mathrm{H}$ atoms using PP-CP (AE) calculations.

In Table II we present the results of calculated dipolar hyperfine couplings. The same data set is reported in Figs. 2(c),(d) for $\mathrm{C}$ and $\mathrm{H}$ atoms, respectively. The largest absolute deviation between our PP calculations and experiment is $23 \mathrm{MHz}$, while the largest deviation for the case of AE calculations is $21 \mathrm{MHz}$. Again PP calculations and AE calculations appear to perform similarly, and are both able to provide reasonably reliable data when compared to experiment.

What we learn from these tests is that, on the one hand, in the case of the Fermi contact term, the inclusion of core polarization in $\mathrm{PP}$ calculations is critical to achieving agreement with AE calculations and with experiment. On the other hand, PP calculations of the dipolar HFI interaction are already in reasonable agreement with $\mathrm{AE}$ data and experiment. In the case of $\mathrm{HCO}$ we observe that the deviation between the calculated isotropic HFI (either PP-CP or AE) and experiment can be rather large, hence care must be used in the interpretation of the calculated HFI parameters. This discrepancy requires further investigation. 
TABLE II: Dipolar hyperfine coupling parameters of small molecules containing $\mathrm{C}$ and $\mathrm{H}$. We report PP calculations, AE calculations, and measured parameters. The atoms are listed according to corresponding chemical formula.

\begin{tabular}{|c|c|c|c|c|c|}
\hline \multirow[t]{2}{*}{ Molecule } & \multirow[t]{2}{*}{ Atom } & \multicolumn{4}{|c|}{ Dipolar HFI (MHz) } \\
\hline & & Axis & $\mathrm{PP}$ & $\mathrm{AE}$ & Exp. \\
\hline \multirow[t]{6}{*}{$\mathrm{CH}_{2} \mathrm{CH}$} & & $b_{x x}$ & -86 & -72 & \\
\hline & $\mathrm{C}(1)$ & $b_{y y}$ & -64 & -56 & \\
\hline & & $b_{z z}$ & 148 & 127 & \\
\hline & & $b_{x x}$ & 37 & 37 & $38^{\mathrm{h}}$ \\
\hline & $\mathrm{H}(3)$ & $b_{y y}$ & -6 & -6 & -11 \\
\hline & & $b_{z z}$ & -32 & -32 & -27 \\
\hline \multirow[t]{12}{*}{$\mathrm{CH}_{2} \mathrm{CH}_{3}$} & & $b_{x x}$ & -83 & -73 & \\
\hline & $\mathrm{C}$ & $b_{y y}$ & -82 & -72 & \\
\hline & & $b_{z z}$ & 165 & 145 & \\
\hline & & $b_{x x}$ & 35 & 38 & $35^{\mathrm{i}}$ \\
\hline & $\mathrm{H}$ & $b_{y y}$ & 2 & 0 & 1 \\
\hline & & $b_{z z}$ & -38 & -38 & -36 \\
\hline & & $b_{x x}$ & -3 & -3 & \\
\hline & $\mathrm{C}$ & $b_{y y}$ & 1 & 1 & \\
\hline & & $b_{z z}$ & 3 & 2 & \\
\hline & & $b_{x x}$ & -4 & -4 & $-3^{\mathrm{i}}$ \\
\hline & $\mathrm{H}$ & $b_{y y}$ & -3 & -3 & -3 \\
\hline & & $b_{z z}$ & 7 & 9 & 6 \\
\hline \multirow[t]{6}{*}{$\mathrm{CH}_{3}$} & & $b_{x x}$ & -70 & -74 & $-63^{1}$ \\
\hline & $\mathrm{C}$ & $b_{y y}$ & -72 & -74 & -63 \\
\hline & & $b_{z z}$ & 150 & 148 & 127 \\
\hline & & $b_{x x}$ & -36 & -39 & $-36^{\mathrm{m}}$ \\
\hline & $\mathrm{H}$ & $b_{y y}$ & -1 & -1 & -1 \\
\hline & & $b_{z z}$ & 35 & 39 & 35 \\
\hline
\end{tabular}

\begin{tabular}{|c|c|c|c|c|c|}
\hline \multirow[t]{2}{*}{ Molecule } & \multirow[t]{2}{*}{ Atom } & \multicolumn{4}{|c|}{ Dipolar HFI (MHz) } \\
\hline & & Axis & $\mathrm{PW}$ & $\mathrm{AE}$ & Exp. \\
\hline \multirow[t]{6}{*}{$\mathrm{H}_{3} \mathrm{CO}$} & & $b_{x x}$ & 7 & 6 & \\
\hline & $\mathrm{C}$ & $b_{y y}$ & 2 & 2 & \\
\hline & & $b_{z z}$ & -9 & -8 & \\
\hline & & $b_{x x}$ & -8 & -7 & \\
\hline & $\mathrm{H}$ & $b_{y y}$ & -4 & -4 & \\
\hline & & $b_{z z}$ & 12 & 12 & \\
\hline \multirow[t]{6}{*}{$\mathrm{HCO}$} & & $b_{x x}$ & -43 & -42 & $-48^{\mathrm{c}}$ \\
\hline & $\mathrm{C}$ & $b_{y y}$ & -33 & -38 & -24 \\
\hline & & $b_{z z}$ & 76 & 80 & 72 \\
\hline & & $b_{x x}$ & -14 & -14 & -14 \\
\hline & $\mathrm{H}$ & $b_{y y}$ & -8 & -7 & -8 \\
\hline & & $b_{z z}$ & 23 & 21 & 23 \\
\hline \multirow[t]{6}{*}{$\mathrm{H}_{2} \mathrm{CO}^{+}$} & & $b_{x x}$ & -25 & -21 & $-15^{\mathrm{k}}$ \\
\hline & $\mathrm{C}$ & $b_{y y}$ & 7 & 6 & 4 \\
\hline & & $b_{z z}$ & 18 & 16 & 10 \\
\hline & & $b_{x x}$ & -10 & -10 & -9 \\
\hline & $\mathrm{H}$ & $b_{y y}$ & -8 & -7 & 5 \\
\hline & & $b_{z z}$ & 18 & 18 & 4 \\
\hline \multirow[t]{3}{*}{$\mathrm{CO}_{3}$} & & $b_{x x}$ & 5 & 4 & $4^{\mathrm{j}}$ \\
\hline & $\mathrm{C}$ & $b_{y y}$ & 5 & 4 & 4 \\
\hline & & $b_{z z}$ & -9 & -9 & -8 \\
\hline
\end{tabular}

${ }^{\mathrm{h}}$ Ref. 39, ${ }^{\mathrm{i}}$ Ref. 40, ${ }^{\mathrm{j}}$ Ref. 33, ${ }^{\mathrm{k}}$ Ref. 36, ${ }^{1}$ Ref. 41, ${ }^{\mathrm{m}}$ Ref. 42, ${ }^{\mathrm{n}}$ Ref. 43.

\section{B. HFI parameters of fullerene derivatives}

In this section we present the HFI parameters calculated for the fullerene derivatives of Fig. 1. Figure 3 shows the calculated spin density distribution for the structures M1 and B1-3 in the triplet state. The spin density is greatest (blue regions) on the fullerene cages, while it is rather small (red regions) on the adducts and the isotopically labeled $\mathrm{C}$ nuclei. As the strength of the HFI scales with the spin density [cf. Eqs. (2), (3)], Fig. 3] suggests that we should expect a rather small HFI strength for all the structures considered.

This observation is consistent with the calculated HFI parameters reported in Table III. which are of the order of only a few $\mathrm{MHz}$. All the pseudopotential calculations in this table include the core polarization correction (PP-CP) discussed in Sec. IIIC For the case of the M1 structure both PP-CP and AE calculations are in good agreement with the experimental data from Ref. 2. In light of the error bars established in Sec. IVA for the test molecules, this level of agreement (around $1 \mathrm{MHz}$ )

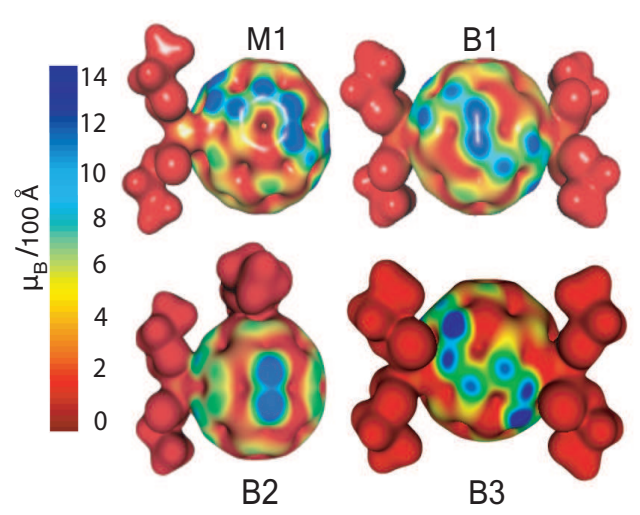

FIG. 3: Spin density distribution in the lowest triplet excited state of the four fullerene derivatives M1 and B1-3 considered in this work. The color code indicates the magnitude of the spin density (the density isosurface corresponds to a isovalue of 0.07 electron $/ \AA^{3}$ ). 


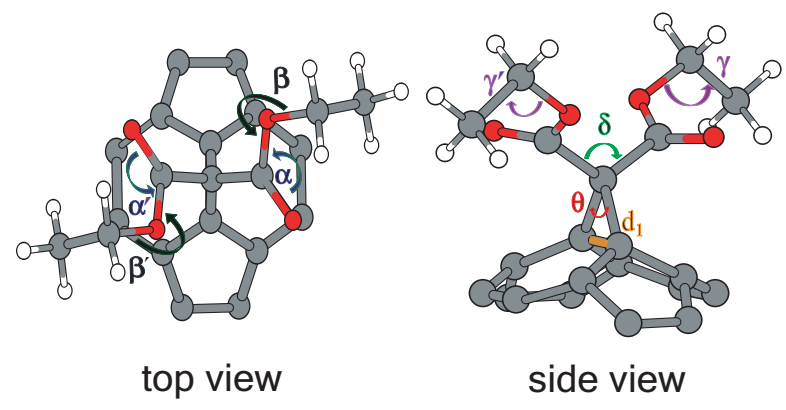

FIG. 4: Ball-and-stick representation of the diethyl malonate adduct on a portion of the $\mathrm{C}_{60}$ fullerene (color code as in Fig. 1). The angles in all the systems considered M1 and B1-3 differ by less than one degree: $\alpha, \alpha^{\prime}=126^{\circ}, \beta, \beta^{\prime}=245^{\circ}$, $\gamma, \gamma^{\prime}=111^{\circ}, \delta=112^{\circ}, \theta=65^{\circ}$. The different $\mathrm{C}-\mathrm{C}$ bond length $d 1$ in the two adducts of B2 lifts the symmetry between the two isotopically labeled nuclei and results into different HFI parameters [cf. B2(1) and B2(2) in Table II].

may be somewhat fortuitous. However it is reasonable to assume that, due to error cancellation, our calculations should provide a reliable description of the relative magnitude of the HFI parameters across various fullerene derivatives. It is possible that accounting for thermal motion may lead to slightly different HFI parameters in our calculations, however we expect that such effects will fall within our error bars established in Sec. IV A. Morover the effect of thermal motion should be similar across the various derivatives, therefore the relative magnitude of the HFI parameters is expected to be reliable.

It is interesting to observe in Table III that the location of the diethyl malonate adduct has a significant effect on the HFI parameters, leading to variations of up to an order of magnitude in the Fermi contact term.

For both the trans-1 isomer B1 and the trans-3 isomer B3 Table III shows that the two ${ }^{13} \mathrm{C}$ nuclei have the same HFI tensor. At variance with this, in the equatorial conformer B2 only one nucleus exhibits a sizeable HFI. In order to rationalize this finding we examine the structural parameters of each fullerene derivative. Figure. 4 shows the geometry of the diethyl malonate adducts on the $\mathrm{C}_{60}$ cage. The structure of the adducts (i.e. bond lengths and bond angles) are almost identical in each fullerene derivative. The only noticeable difference is that the length of the $\mathrm{C}-\mathrm{C}$ bond bridging the adduct to the fullerene is $1.58 \AA$ for both adducts in the structures B1 and B3, while in the structure B2 the two adducts have different C-C bond lengths of 1.60 and $1.57 \AA$, respectively. This indicates that in the equatorial conformer B2 one of the two nuclear spins is closer than the other to the $\mathrm{C}_{60}$ cage. Since the spin density is largest on the fullerene cage, the nuclear spin with the shorter $\mathrm{C}-\mathrm{C}$ bond length can be expected to exhibit a larger HFI strength, consistent with the results in Table III.

In Ref. 2 it was shown that, in systems like the structures B1-3 considered here, it should be possible to
TABLE III: Calculated HFI parameters of the fullerene derivatives M1 and B1-3 shown in Fig. 11 We present data from pseudopotential calculations including core polarization correction (PP-CP), and we also provide the calculated Fermi contact term without core polarization correction (PP) for completeness. The dipolar terms are the same in both cases. For the fullerene mono-adduct M1 we also provide AE data and experimental data from Ref. 2 (measured at $20 \mathrm{~K}$ ) for comparison. In the case of the B1-3 systems the values for the two ${ }^{13} \mathrm{C}$-labeled nuclei are reported as (1) and (2), respectively. All the data are in $\mathrm{MHz}$.

\begin{tabular}{lrrrr}
\hline \hline Structure & $a$ & $\mathrm{~b}_{x x}$ & $\mathrm{~b}_{y y}$ & $\mathrm{~b}_{z z}$ \\
\hline $\mathrm{M} 1, \mathrm{PP}-\mathrm{CP}$ & 2.0 & -1.6 & -2.3 & 4.0 \\
$\mathrm{M} 1, \mathrm{PP}$ & 4.1 & & & \\
$\mathrm{M} 1, \mathrm{AE}$ & 1.0 & -1.3 & -2.0 & 3.4 \\
$\mathrm{M} 1, \mathrm{Expt}$ & 2.0 & & & \\
\hline $\mathrm{B} 1(1), \mathrm{PP}-\mathrm{CP}$ & 1.0 & -0.7 & -1.2 & 1.9 \\
$\mathrm{~B} 1(1), \mathrm{PP}$ & 2.2 & & & \\
$\mathrm{~B} 1(2), \mathrm{PP}-\mathrm{CP}$ & 1.0 & -0.7 & -1.2 & 1.9 \\
$\mathrm{~B} 1(2), \mathrm{PP}$ & 2.2 & & & \\
\hline $\mathrm{B} 2(1), \mathrm{PP}-\mathrm{CP}$ & 0.2 & 0.0 & 0.6 & -0.6 \\
$\mathrm{~B} 2(1), \mathrm{PP}$ & 0.3 & & & \\
$\mathrm{~B} 2(2), \mathrm{PP}-\mathrm{CP}$ & 1.5 & -2.3 & -2.4 & 4.7 \\
$\mathrm{~B} 2(2), \mathrm{PP}$ & 4.4 & & & \\
\hline $\mathrm{B} 3(1), \mathrm{PP}-\mathrm{CP}$ & 2.2 & -1.2 & -1.8 & 3.1 \\
$\mathrm{~B} 3(1), \mathrm{PP}$ & 3.9 & & & \\
$\mathrm{~B} 3(2), \mathrm{PP}-\mathrm{CP}$ & 2.2 & -1.2 & -1.8 & 3.1 \\
$\mathrm{~B} 3(2), \mathrm{PP}$ & 3.9 & & & \\
\hline \hline
\end{tabular}

achieve the entanglement of the two nuclear spins if the following conditions are fulfilled: (i) the Fermi contact interaction when the system is photo-excited to its triplet state is in the range $2-16 \mathrm{MHz}$. (ii) the two isotopically labeled $\mathrm{C}$ nuclei exhibit a very similar strength of the HFI. Table III shows that, on the one hand, the trans-1 B1 and the equatorial B2 isomers do not fulfill at least one of these requirements. On the other hand, the trans-3 B3 isomer fulfills both criteria, and hence it represents a promising candidate for nuclear spin entanglement. This finding is particularly interesting since the B3 structure can be produced with the relatively high yield of $12 \%$ (cf. Sec. III B).

\section{CONCLUSIONS}

In this work we perform calculations of the hyperfine couling tensor for photo-excited fullerene derivatives of interest in spin-based quantum information processing. We report extensive validation of pseudopotential methods against all-electron calculations and experimental data for a series of small radicals. A key observation is that PP calculations can deliver an accuracy comparable to AE calculations, provided the effect of core polariza- 
tion is included.

We investigate the HFI strength in a series of photoexcited diethyl malonate fullerene derivates, which are potential candidates for nuclear spin entanglement operations. We clarify the relation between the calculated HFI parameters and the underlying atomic-scale structure of these systems. We identify one particular structure, the trans-3 isomer B3, as the best match to the entanglement criteria set in Ref. 2. The fullerene derivative thus identified can be produced with relatively high yield and thus represents a potential candidate for achieving the entanglement of two nuclear spins in a photo-excited chromophore.

Our work provides a set of validation tests and benchmarks for future calculations of HFI parameters using pseudopotential methods. In addition, this work represents a first step towards the synergistic use of atomistic modelling and electron spin resonance experiments in the design of functional materials for quantum information technology.

\section{Acknowledgments}

We thank K. Porphyrakis, A. Ardavan and G. A. Briggs for stimulating discussions. The calculations were performed at the Oxford Supercomputing Centre. The spin density images were rendered using Gabedit 2.3.0. $\stackrel{46}{ }$ We acknowledge support from the European Research Council under the European Community's Seventh Framework Programme (FP7/2007-2013) / ERC grant agreements no. 239578 (F.G.) and 279781 (J.J.L.M.). JJLM thanks the EPSRC (EP/I035536/1), the Royal Society, and St. John's College Oxford.
1 J. J. L. Morton, A. M. Tyryshkin, R. M. Brown, S. Shankar, B. W. Lovett, A. Ardavan, T. Schenkel, E. E. Haller, J. W. Ager, and S. A. Lyon, Nature 455, 1085 (2008).

2 M. Schaffry, V. Filidou, S. D. Karlen, E. M. Gauger, S. C. Benjamin, H. L. Anderson, A. Ardavan, G. A. D. Briggs, K. Maeda, K. B. Henbest, F. Giustino, J. J. L. Morton, and B. W. Lovett, Phys. Rev. Lett. 104, 200501 (2010).

${ }^{3}$ E. Zurek, C. J. Pickard, and J. Autschbach, J. Phys. Chem. A 113, 4117 (2009).

4 M. d'Avezac, N. Marzari, and F. Mauri, Phys. Rev. B 76, 165122 (2007).

${ }^{5}$ L. Shao, J. R. Yates, and J. Titman, J. Phys. Chem. A 111, 13126 (2007).

6 F. Mauri, B. G. Pfrommer, and S. G. Louie, Phys. Rev. Lett. 77, 5300 (1996).

7 L. Truflandier, M. Paris, and F. Boucher, Phys. Rev. B 76, 035102 (2007).

8 S. Rossano, F. Mauri, C. J. Pickard, and I. Farnan, J. Phys. Chem. B 109, 7245 (2005).

${ }^{9}$ C. J. Pickard and F. Mauri, Phys. Rev. Lett. 88, 086403 (2002).

10 S. A. Joyce, J. R. Yates, C. J. Pickard, and S. P. Brown, J. Am. Chem. Soc. 130, 12663 (2008).

11 U. Gerstmann, M. Rohrmuller, F. Mauri, and W. G. Schmidt, Physica Status Solidi 7, 157 (2009).

12 U. Gerstmann, A. P. Seitsonen, D. Ceresoli, F. Mauri, H. J. von Bardeleben, J. L. Cantin, and J. Garcia Lopez, Phys. Rev. B 81, 195208 (2010).

13 A.J. Hoff, in Advanced EPR: Application in Biology and Biochemistry, (Elsevier, Amsterdam, 1989).

14 L. van Keand and M. K. Bowman, in Modern Pulsed and Continuous Wave Electron Spin Resonance, (Wiley, New York, 1990).

15 P. E. Blöchl, Phys. Rev. B 50, 17953 (1994)

16 C. Van de Walle and P. E. Blöchl, Phys. Rev. B 47, 4244 (1993).

17 A. Hirsch, I. Lamparth, and H. Karfunkel, Angewandte Chemie. 33, 437 (1994).

18 J. P. Perdew, K. Burke, and M. Ernzerhof, Phys. Rev.
Lett. 77, 3865 (1996).

19 N. Troullier and J. L. Martins, Phys. Rev. B 43, 1993 (1991).

20 M. Fuchs and M. Scheffler, Comput. Phys. Comm. 119, 67 (1999).

21 C. Pickard and F. Mauri, Phys. Rev. B 63, 245101 (2001).

22 A. J. Freeman and R. E. Watson, Phys. Rev. Lett. 5, 489 (1960).

${ }^{23}$ R. E. Watson and A. J. Freeman, Phys. Rev. 123, 2027 (1961).

24 M. S. Barhamy, M. H. F. Sluiter, and Y. Kawazoe, Phys. Rev. B 76, 035124 (2007).

${ }^{25}$ P. Giannozzi, S. Baroni, N. Bonini, M. Calandra, R. Car, C. Cavazzoni, D. Ceresoli, G.L. Chiarotti, M. Cococcioni, I. Dabo, A. Dal Corso, S. Fabris, G. Fratesi, S. de Gironcoli, R. Gebauer, U. Gerstmann, C. Gougoussis, A. Kokalj, M. Lazzeri, L. Martin-Samos, N. Marzari, F. Mauri, R. Mazzarello, S. Paolini, A. Pasquarello, L. Paulatto, C. Sbraccia, S. Scandolo, G. Sclauzero, A. P. Seitsonen, A. Smogunov, P. Umari, and R. M. Wentzcovitch, J. Phys. Condens. Matter 21, 395502 (2009); http://www.quantum-espresso.org

26 Gaussian 09, Revision A.1, M. J. Frisch, G. W. Trucks, H. B. Schlegel, G. E. Scuseria, M. A. Robb, J. R. Cheeseman, G. Scalmani, V. Barone, B. Mennucci, G. A. Petersson, H. Nakatsuji, M. Caricato, X. Li, H. P. Hratchian, A. F. Izmaylov, J. Bloino, G. Zheng, J. L. Sonnenberg, M. Hada, M. Ehara, K. Toyota, R. Fukuda, J. Hasegawa, M. Ishida, T. Nakajima, Y. Honda, O. Kitao, H. Nakai, T. Vreven, J. A. Montgomery, Jr., J. E. Peralta, F. Ogliaro, M. Bearpark, J. J. Heyd, E. Brothers, K. N. Kudin, V. N. Staroverov, R. Kobayashi, J. Normand, K. Raghavachari, A. Rendell, J. C. Burant, S. S. Iyengar, J. Tomasi, M. Cossi, N. Rega, J. M. Millam, M. Klene, J. E. Knox, J. B. Cross, V. Bakken, C. Adamo, J. Jaramillo, R. Gomperts, R. E. Stratmann, O. Yazyev, A. J. Austin, R. Cammi, C. Pomelli, J. W. Ochterski, R. L. Martin, K. Morokuma, V. G. Zakrzewski, G. A. Voth, P. Salvador, J. J. Dannenberg, S. Dapprich, A. D. Daniels, Farkas, J. B. Foresman, J. V. Ortiz, J. Cioslowski, and D. J. Fox, Gaussian, Inc., 
Wallingford CT, 2009.

27 V. Barone, in Recent Advances in Density Functional Methods, (World Scientific, Singapore, 1996), Part I.

28 O. Gunnarsson and B. I. Lundqvist, Phys. Rev. B 13, 4274 (1976).

29 Y. Kanai and J. C. Grossman, Nano Letters 7, 1967 (2007).

${ }^{30}$ R. W. Fessenden and R. H. Schuler, J. Chem. Phys. 39, 2147 (1963).

31 R. W. Fessenden, J. Phys. Chem. 71, 74 (1967).

32 D. W. Ovenall and D. H. Whiffen, Mol. Phys. 4, 135 (1961).

33 G. W. Chantry, A. Horsfield, J. R. Morton, and D. H. Whiffen, Mol. Phys. 5, 589 (1962).

34 T. Momose, Y. Endo, E. Hirota, and T. Shida, J. Chem. Phys. 88, 5338 (1988).

35 H. Nakatsuji, and M. Izawa, J. Chem. Phys. 91, 6205 (1989).

36 R. W. Holmberg, J. Chem. Phys. 51, 3255 (1969).
37 L. B. Knight and J. Steadman, J. Chem. Phys. 88, 5338 (1988).

38 D. M. Chipman, Theor. Chim. Acta. 82, 93 (1992).

39 M. Iwasaki, B. Eda, and K. Toriyama, J. Chem. Phys. 52, 3837 (1970).

40 T. Shiga, H. Yamaoka, and A. Lund, Z. Naturforsch. 29a, 653 (1974).

41 L. B. Knight and J. Steadman, J. Chem. Phys. 80, 1018 (1984).

42 R. W. Fessenden, J. Phys. Chem. 71, 74 (1967).

43 J. Janecka, H. M. Vyas, and M. Fujimoto, J. Chem. Phys. 54, 3229 (1971).

44 D. J. van den Heuvel et al., Chem. Phys. Lett. 23, 181 (1994).

45 A. Sassara, G. Zerza, and M. Chergui, Chem. Phys. Lett. 261, 213 (1996).

46 A. Alouche, http://gabedit.sourceforce.net/ 